\title{
Terapia antirretroviral directamente observada en mujeres privadas de libertad
}

\author{
L De Carolis, C Wainstein
}

Instituto Correccional de Mujeres, Unidad No 3, Servicio Penitenciario Federal, Argentina.

\section{RESUMEN}

Objetivos: La adherencia al tratamiento antirretroviral de alta eficacia (TARV) se considera el gold standard para el éxito del mismo. Para mejorar la adherencia en persona privadas de libertad viviendo con VIH-Sida, se propuso la utilización del tratamiento directamente observado tomando el modelo para el tratamiento de la tuberculosis.

Material y métodos: se incluyeron las mujeres VIH positivas con criterios de TARV que voluntariamente decidieron participar. Los datos principales a evaluar fueron control inicial y final de CD4 y carga viral para VIH.

Resultados: estudiamos 52 mujeres, edad promedio 34 años, entre 1 y 20 años de infección VIH. CD4 menor a 100 cél/ml inicial en 16 pacientes $(30.7 \%$ ) y final en 4 pacientes $(7.6 \%)$. Carga viral indetectable inicial en ninguna paciente y final en 33 pacientes $(63.4 \%) 21 \%$ con infecciones oportunistas, principal tuberculosis. 17\% coinfectadas con HCV. El esquema de TARV más utilizado fue 2 INTR más 1 INNTR.

Conclusiones: La estrategia DOT aplicada al TARV fue efectiva en nuestras pacientes, evidenciado por el aumento de los CD4 y el mayor número de pacientes con disminución de la carga viral hasta permanecer indetectables. Si bien es difícil de implementar en tratamientos crónicos consideramos que es una herramienta útil para personas privadas de libertad.

Palabras clave: Seroprevalencia de VIH; Terapia Antirretrioviral Altamente Activa; Prisiones; Mujeres; Agentes Anti VIH.

\section{DIRECTLY OBSERVED ANTI-RETROVIRAL THERAPY AMONGST FEMALE INMATES}

\begin{abstract}
Objectives: It is considered that the gold standard for success in HAART is adherence. To improve adherence amongst inmates with HIV-AIDS, the use of directly observed treatment (DOT) is proposed using the tuberculosis treatment model.

Material and methods: HIV positive female patients with ARVT criteria who voluntarily participated were used for the study. The initial and final CD4 cell count and HIV viral load were the principal data used for assessment purposes.

Results: 52 women with an average age of 34 years were studied, with an average HIV infection time span of between 1 and 20 years. Initial CD 4 cell count of $<100$ copies $/ \mathrm{mL}$ in 16 patients $(30.7 \%)$ and an equivalent final count in 4 patients $(7.6 \%)$ were found. Initial undetectable viral loads were not found in any patient, while final undetectable viral loads were found in 33 (63.4\%). 21\% of patients had opportunistic infections. The most important of these was tuberculosis, followed by HCV co-infection. The most frequently used ARVT schedule was two NRTI with one NNTRI.

Conclusions: The application of DOT strategy to ARVT was effective amongst our patients, as shown by the increase in CD4 counts and the increased number of patients with reductions in viral loads to undetectable levels. While it is a tool that is not easy to use for cases of chronic treatment, we do consider it to be useful for prison inmates.
\end{abstract}

Key words: HIV Seroprevalence; Antiretroviral Therapy, Highly Active; Prisons; Women; Anti-HIV Agents.

Texto recibido: mayo 2009

Texto aceptado: septiembre 2009 


\section{INTRODUCCIÓN}

El tratamiento de la infección por HIV o del SIDA enfermedad es actualmente una indicación de por vida, por lo tanto la adherencia al esquema instituido es esencial en la evolución es decir para la reducción de la morbi-mortalidad. En los últimos años hemos asistido a los beneficios extraordinarios de la terapia antirretroviral (TARV), sin embargo, el entusiasmo generado por estos éxitos recientes se ha visto opacado por factores de origen biológicos, clínicos, sociales y económicos que limitan el acceso a los tratamientos y su éxito terapéutico.

Hoy día se acepta que la adherencia al tratamiento tiene igual importancia que la potencia del tratamiento en sí mismo.

Es por ello que se han implementado intervenciones para promover la adherencia al TARV, que comprenden:

Intervenciones con el paciente, es decir generarle rutinas que integren sus medicamentos a sus actividades cotidianas. Acá se incluye la estrategia DOT o TDO (Tratamiento Directamente Observado), el cual se ha utilizado con éxito en poblaciones privadas de la libertad u otros grupos pero es complejo de implementar en otras poblaciones.

Intervenciones hacia el equipo de salud, comprenden estrategias de entrevistas periódicas con el paciente para establecer un medio apropiado que genere y determine el momento en que el paciente está en condiciones de comenzar su tratamiento. A partir de allí el medico tratante deberá realizar la evaluación periódica de la adherencia, esencial para mantener esta conducta e ir identificando obstáculos para poder subsanarlos. Ninguna de estas intervenciones es suficiente por sí sola.

El DOTS es la estrategia recomendada internacionalmente para asegurar la curación de la tuberculosis ${ }^{1}$. Se basa en cinco principios clave que son comunes a las estrategias de control de enfermedades y se apoya, para frenar la diseminación de la tuberculosis, en el diagnóstico precoz y la curación de los casos contagiosos, esta última estrategia para prevenir la transmisión y controlar la tuberculosis fue destacado por Crofton a comienzos de 1960.

Surge así el término "tratamiento directamente observado" el cual estuvo en uso durante varios años hasta que fuera modificado por la OMS a "tratamiento acortado directamente observado" en 1995, y empleado para designar a una estrategia completa para el control de la tuberculosis.

La observación directa del tratamiento en la cual "una persona entrenada y supervisada observa al pa- ciente mientras este ingiere los comprimidos" es fundamental en la estrategia DOTS para garantizar la adherencia al tratamiento ${ }^{2}$. Los primeros documentos de la OMS recalcaban la importancia de la observación directa realizada por personal sanitario ${ }^{3}$. Más tarde, la experiencia lograda con los programas DOTS alrededor del mundo demostró que las personas legas entrenadas fueron, por lo menos, igualmente eficaces para observar el tratamiento $0^{4-7}$.

Sin embargo la aplicación del DOT en el TARV tiene algunos puntos distintos dependiendo de las patologías en si mismo, a saber:

- La tuberculosis (TB) es generalmente una enfermedad curable, la infección por HIV es controlable.

- En TB el tratamiento tiene una duración limitada, en infección por HIV al momento la terapia es de por vida.

- El cumplimiento del tratamiento de la TB se considera un tema de salud pública y por lo tanto se puede inducir al paciente al cumplimiento del mismo, el TARV es voluntario y decisión del propio paciente.

\section{MATERIAL Y MÉTODOS}

Se incluyeron las mujeres con infección por HIV alojadas en el Instituto Correccional de Mujeres, Unidad $N^{\circ} 3$ del Servicio Penitenciario Federal, con criterios para recibir TARV según pautas nacionales.

Para el inicio del TARV realizaron consultas con el medico tratante, apoyo psicológico a demanda y fueron instruidas sobre la entrega diaria de los medicamentos y la toma del mismo frente al personal del equipo de salud.

El personal encargado de la administración de los medicamentos se seleccionó entre el personal del equipo de salud, enfermería, perteneciente al personal de seguridad dado que son los únicos autorizados para la entrega de medicamentos dentro del penal.

Dichas personas fueron capacitadas previamente sobre infección por HIV-Sida, importancia de la adherencia al tratamiento y esquema DOT o TDO.

Se evaluaron los siguientes datos: edad, años de infección VIH, comorbilidades, esquema de tratamiento antirretroviral, valores de CD4 y de Carga viral para HIV al inicio y al final del programa.

\section{RESULTADOS}

Actualmente, en la Unidad mencionada se encuentran alojadas 461 internas, 27 de ellas son HIV 
positivas $(5,85 \%)$. Durante el periodo en estudio participaron 91 mujeres HIV positivas, 52 con criterio para ser medicadas y con consentimiento para recibirla así como el TDO.

El rango de edad estuvo entre 21 y 54 años, el promedio fue de $34+/-7$ años.

El año de diagnostico de infección de las pacientes estaba entre 1988 y 2007, es decir entre 21 y 1 año de diagnóstico, el $78 \%$ tenia 10 o menos años de infección.

El valor de CD4 inicial estuvo en un rango entre 19 y 868 células $/ \mathrm{mm}^{3}$. El valor elevado de algunas pacientes, mayor de 500 células $/ \mathrm{mm}^{3}$, se debió a que ingresaron al penal con diagnóstico y tratamiento en curso, de todos modos se incorporaron al TDO.

El valor del CD4 inicial era de menos de 100 células $/ \mathrm{mm}^{3}$ en 16 pacientes $(30.7 \%$ ) de las pacientes, mientras que dicho valor se presentó al final del estudio en 4 pacientes $(7.6 \%)$.

Los resultados finales fueron para valores de CD4 4 pacientes con CD4 menor a 100 células $/ \mathrm{mm}^{3}, 14$ entre 99 y 199 células $/ \mathrm{mm}^{3}$ y 30 con más 300 células. (Gráfico 1).

La carga viral $(\mathrm{CV})$ inicial presentaba un rango entre 101 y 514.000 , ninguna paciente presentaba una $\mathrm{CV}$ indetectable; los valores más bajos, entre 50 y 5000 copias correspondieron a 11 pacientes $(21.1 \%)$, mientras que 20 de ellas $(38.4 \%)$ tenían más de 500.000 copias.

El último control mostró que 33 pacientes (63.4\%) tenían CV indetectable ( $<50$ copias $/ \mathrm{ml}) ; 7$ pacientes tenían menos de 5.000 copias y en el resto el valor era mayor que el antedicho. (Gráfico 2) Del total de pacientes indetectables $20(60 \%)$ tenía 5 años o menos de diagnóstico de la infección.

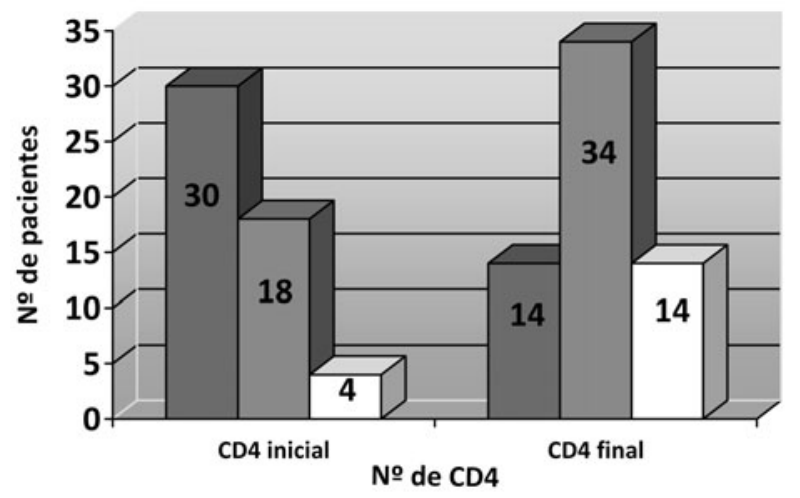

0 a $199 \square 200$ a $499 \square$ mayor a 500

Gráfico 1: comparación entre el recuento de CD4 antes de iniciado y después de finalizado el período de TDO.
La presencia de infecciones oportunistas se observó en 11 que representaron el 21\%, siendo la más importante la tuberculosis, la cual se presentó en 8 de las 11 pacientes sintomáticas (81\%).

La coinfección con virus de la Hepatitis C (HCV) se presentó en 9 casos.

El esquema de tratamiento recibido era 2 INTR +1 INNTR en 40 pacientes, 2 INTR + 1 IP en 2 casos, IP/r + 2INTR (o 1 INuTR + 1 INTR o 3 INTR o 1 INNTR + 2 INTR) en 10 pacientes y 3 INTR en 1 caso.

El tiempo de tratamiento fue entre 1 y 4 años.

\section{DISCUSIÓN}

El tratamiento directamente observado (TDO) es otra estrategia para mejorar la adherencia. Ha sido empleado con éxito en el tratamiento de la tuberculosis y se han hecho intentos de adaptarlo a las características del TARV.

Diversos estudios han proporcionado resultados preliminares favorables del TDO en el TARV, a corto y largo plazo ${ }^{8-10}$, pero aún no están bien definidos los programas que mejor se adaptan al mismo, siendo necesario, además, clarificar la duración óptima, cuales son los mejores candidatos para ingresar en estos programas y si su relación coste-efectividad.

Las estrategias de intervención individualizadas, basadas en estrategias psico-educativas y de asesoramiento personal, con capacidad para adaptar el esquema del TARV a los hábitos de vida del paciente y proporcionando estrategias de resolución de problemas, han demostrado ser eficaces para incrementar la adherencia al tratamiento y mejorar la respuesta del mismo.

El tratamiento directamente observado (TDO) no puede recomendarse de forma generalizada. Sin

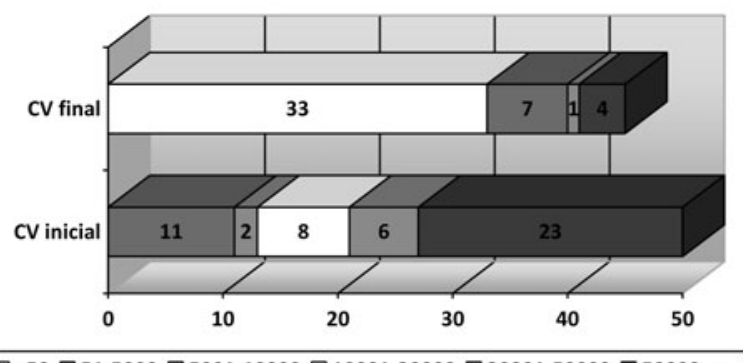

$\square<50 \square 51-5000 \square 5001-10000 \square 10001-30000 \square 30001-50000 \square 500000+$

Gráfico 2: Comparación entre las cargas virales antes de iniciar y al finalizar el TDO. 
embargo, puede tener interés y ser efectivo en pacientes con situaciones especiales (reclusos, marginación social extrema y programas de mantenimiento con metadona $)^{11}$.

Recientemente se han producido avances en el TARV que ha implicado la posibilidad de realizar tratamientos sencillos de alta potencia que favorecen de manera muy importante la adherencia al tratamiento. Estos avances son la coformulación de varios principios activos en una sola forma de dosificación, la disponibilidad de fármacos que pueden administrarse una vez al día y por consiguiente, la posibilidad de combinaciones en dosis única diaria ${ }^{12}$.

\section{CONCLUSIONES}

La adherencia al TARV tiene un papel fundamental en la decisión de iniciar el TARV y en la duración de la respuesta virológica, habiéndose demostrado que la falta de cumplimiento es la principal causa de fracaso terapéutico. La falta de adherencia se correlaciona positivamente con el aumento de los ingresos hospitalarios, la evolución a SIDA y la mortalidad del paciente con infección por HIV, siendo además por su elevado coste, una causa de ineficiencia de los recursos públicos.

El TARV debe individualizarse y adaptarse a las necesidades y preferencias de cada paciente. Son aconsejables las pautas más sencillas en cuanto a número de comprimidos y a dosis diarias.

En pacientes con supresión virológica mantenida, alcanzada con una pauta compleja, puede considerarse simplificar el TARV a pautas que hayan demostrado igual o mejor seguridad y eficacia.

Los factores que han demostrado estar relacionados con la adherencia de una forma más relevante incluyen la complejidad del tratamiento, los efectos secundarios, los problemas psicológicos, la adicción activa a drogas y/o alcohol, la falta de soporte social y familiar y las actitudes y creencias del paciente acerca del tratamiento.

Se deben utilizar varios métodos asociados para la estimación de la adherencia. En el contexto de seguimiento rutinario de la adherencia, deben utilizarse métodos factibles, adaptados a la realidad del hospital y lo más universalmente aplicables. Puede considerarse un mínimo aceptable la asociación de un cuestionario validado y el registro de dispensación del Servicio de Farmacia.

En los pacientes en los que no se alcancen niveles de adherencia o cumplimiento considerados co- mo adecuados, se deben intentar estrategias de intervención, de forma interdisciplinaria, basadas en aspectos educativos y psicosociales, con asesoramiento personal y con capacidad para adaptar el esquema del TARV a los hábitos de vida del paciente y proporcionando estrategias de resolución de problemas.

Actualmente los esquemas más sencillos en cuanto a número de comprimidos y a dosis diarias se pueden considerar como herramienta para simplificar el TARV.

En este marco se encuadra la estrategia TDO para ser utilizada en el TARV, donde si bien no hay demasiada experiencia por las diferencias entre las patologías en las que se utiliza, tuberculosis y HIV, la aplicación en ciertos y determinados ámbitos como ser las prisiones, puede ser una eficaz ayuda en el momento de lograr mejor evolución con mejor calidad de vida para las personas viviendo con HIV privadas de libertad.

\section{CORRESPONDENCIA}

Dra. Celia Wainstein

Honorio Pueyrredon, 364, 8 A

1405 Ciudad de Buenos Aires

Argentina

Email: wainsteincelia@gmail.com

\section{REFERENCIAS BIBLIOGRAFICAS}

1. What is DOTS? A guide to understanding the WHO-recommended TB control strategy known as DOTS. Geneva, World Health Organization, 1999 (document WHO/CDS/CPC/ TB/99.270).

2. Walley JD, et al. Effectiveness of the direct observation component of DOTS for tuberculosis: a randomised controlled trial in Pakistan. Lancet, 2001, 357: 664-669.

3. Becx-Bleumink M, et al. High cure rates in smear-positive tuberculosis patients using ambulatory treatment with once-weekly supervision during the intensive phase in Sulawesi, Republic of Indonesia. International Journal of Tuberculosis and Lung Disease, 1999, 3: 1066-1072.

4. Farmer P, Kim JY. Community based approaches to the control of multidrug-resistant tuberculosis: introducing "DOTS-plus". British Medical Journal, 1998, 317:671-674. 
5. Global tuberculosis control. WHO Report 2003. Geneva, World Health Organization, 2003 (document WHO/CDS/TB/2003.316).

6. Global tuberculosis programme. Report of the ad hoc committee on the tuberculosis epidemic. Geneva, World Health Organization, 1998 (document WHO/TB/98.245).

7. Global DOTS expansion plan: progress in TB control in high-burden countries. Geneva, World Health Organization, 2001 (document WHO/ CDS/STB/2001.11).

8. Conway B, Prasad J, Reynolds R, Farley J, Jones $\mathrm{M}$, Jutha S, et al. Directly observed therapy for the management of HIV-infected patients in a methadone program. Clin Infect Dis 2004; 38 Suppl 5: S402-8.

9. Macalino GE, Mitty JA, Bazerman LB, Singh K, McKenzie M, Flanigan T. Modified directly ob- served therapy for the treatment of HIV-seropositive substance users: lessons learned from a pilot study. Clin Infect Dis 2004; 38 Suppl 5: S393-7.

10. Mitty JA, Stone VE, Sands M, Macalino G, Flanigan T. Directly observed therapy for the treatment of people with human immunodeficiency virus infection: a work in progress. Clin Infect Dis 2002; 34(7): 984-90.

11. De Carolis L, Wainstein C. Directly Observed Antiretroviral Therapy in HIV Positive Inmate Women in Buenos Aires, Argentina. The first IAS conference on HIV Pathogenesis and treatment, July 8-11 Buenos Aires, Argentina, 2001. Poster 428.

12. Knobel H, Escobar I, Ortega L, Martínez Conde MT, Casado JL, et al. Recomendaciones GESIDA/SEFH/PNS para mejorar la adherencia al tratamiento antirretroviral en el año 2004. 